\title{
Spontaneous Perforation of Sigmoid Colon Due to Chronic Constipation
}

\author{
Tomo Matsumoto ${ }^{1}$, Hidejiro Kawahara ${ }^{1}$,Yuki Hiramoto ${ }^{1}$, Mitsumasa Takeda ${ }^{1}$, Takeyuki Misawa ${ }^{1}$ \\ and Katsuhiko Yanaga ${ }^{2}$
}

'Department of Surgery, Kashiwa Hospital, Jikei University School of Medicine, Chiba, Japan 2Department of Surgery, Jikei University School of Medicine, Tokyo, Japan

\section{ABSTRACT}

Background/Aim: Spontaneous perforation of the sigmoid colon is defined as a sudden perforation of the normal colon in the absence of diseases. However, its etiology remains unclear.

Methods: Between January 2010 and December 2017, twenty-three patients who underwent Hartmann's operative procedure with colostomy for acute peritonitis due to sudden perforation of the sigmoid colon at our hospital were enrolled in this study. Patients were divided into two groups according to the cause of the perforation (i.e., spontaneous perforation in seven and diverticulosis in sixteen).

Results: All patients had a long history of chronic constipation. The mean age of the spontaneous group was $79.0(71-87)$ years, which was significantly older than that of the diverticulosis group. Significant differences in gender, complication rates, or nutritional factors, such as body mass index, serum total protein, albumin, and cholinesterase, were not identified between the two groups. Although the lymphocyte counts of the spontaneous group were significantly lower than those of the diverticulosis group $(p=0.029)$, significant differences were not identified in the neutrophil-to-lymphocyte ratio between the two groups. The diameters of the perforations in the spontaneous group were significantly larger than that of the diverticulosis group $(p=0.046)$.

Conclusion: Spontaneous perforation of the sigmoid colon due to chronic constipation may not be due to undernutrition but instead due to immune depression.

Key words: Spontaneous perforation, constipation, sigmoid colon, surgery

\section{INTRODUCTION}

Spontaneous perforation of the colon is defined as a sudden perforation of the normal colon without diseases, such as tumors, diverticulosis or external injury (1). The disease has often been seen in patients having a long history of chronic constipation. The solid feculent mass compresses the colonic wall, diminishes the blood supply and leads to ischemia and necrosis of the colonic mucosa, which can cause marked changes in feculent ulcer formation. Ulcers may lead to colonic rupture in some cases (2-5). However, the etiology of colonic

\author{
Corresponding author: \\ Tomo Matsumoto, MD \\ Department of Surgery \\ Kashiwa Hospital, Jikei University \\ School of Medicine, 163-1 \\ Kashiwashita, Kashiwashi, Chiba \\ 277-8567, Japan \\ Tel: +81471641111 Ext. 3421 \\ Fax: +81471633488 \\ E-mail: kawahide@jikei.ac.jp
}


rupture remains unclear. The aim of this study is to examine the etiology of spontaneous perforation of the sigmoid colon.

\section{PATIENTS AND METHODS}

The Ethics Committee for Biomedical Research of the Jikei Institutional Review Board approved the protocol [30-221 (9242)], and all patients or their family members provided their written informed consent to participation.

Between January 2010 and December 2017, twentythree patients (ten males and thirteen females) who underwent Hartmann's operative procedure with colostomy for acute peritonitis due to sudden perforation of the sigmoid colon at our hospital were enrolled in this study. Patients were divided into two groups according to the cause of the perforation, which was spontaneous in seven patients and diverticulosis in sixteen patients. All patients had a long history of chronic constipation. Body weight and hematological/ biochemical parameters were investigated to assess the nutritional status of the patients. The hematological/ biochemical parameters included levels of total protein, albumin, Cholinesterase (ChE), and the lymphocyte count, which were measured before surgery. Body mass index (BMI) was calculated using the standard formula: weight $(\mathrm{kg}) /$ height $\left(\mathrm{m}^{2}\right)$.

\section{Statistical analysis}

Continuous variables are expressed as the means and ranges. The Wilcoxon rank-sum test was used to compare the continuous variables, and the chi-square test was used to compare the categorical data. A p-value of less than 0.05 was used to indicate significance. All data were analyzed using the Statistical Package for Social Sciences (SPSS) 24.0, (IBM SPSS, Tokyo, Japan).

\section{RESULTS}

The mean age of the spontaneous group was 79.0 (71-87) years, which was significantly older than that of the diverticulosis group. Significant differences in gender, complication rates, and nutritional factors, such as body mass index, serum total protein, albumin, and cholinesterase, were not identified between the two groups. Although the lymphocyte counts of the spontaneous group were significantly lower than those of the diverticulosis group $(p=0.029)$, significant differences were not identified in the neutrophil-to-lymphocyte ratio between the two groups. The diameters of the perforations of the spontaneous group were significantly larger than those of the diverticulosis group $(p=0.046)$. The average length of stay in the postoperative intensive care unit (ICU) was significantly longer for the spontaneous group than for the diverticulosis group $(p=0.022)$ (table 1$)$.

\section{DISCUSSION}

Generally, colonic perforation is most often caused by feces. Maurer at al.(3) proposed the diagnostic criteria for feculent colonic perforation: (1) The perforation is a

Table 1 - Clinicopathological features of the patients

\begin{tabular}{|c|c|c|c|}
\hline Characteristic & Spontaneous $(n=7)$ & Diverticulosis $(n=16)$ & $\mathrm{p}$ value \\
\hline Mean age (range), years & $79.0(71-87)$ & $70.6(44-85)$ & 0.042 \\
\hline Gender, n (\%) & & & 0,867 \\
\hline Male & $3(43)$ & $7(44)$ & \\
\hline Female & $4(57)$ & $9(56)$ & \\
\hline Complication, $\mathrm{n}(\%)$ & & & 0.740 \\
\hline Heart disease & $0(0)$ & $1(6)$ & \\
\hline Lung disease & $0(0)$ & $1(6)$ & \\
\hline Renal disease & $0(0)$ & $1(6)$ & \\
\hline Other & $0(0)$ & $1(6)$ & \\
\hline Body mass index, mean (range) $\mathrm{kg} / \mathrm{m}^{2}$ & $21.2(18.1-23.0)$ & $21.9(18.8-26.1)$ & 0.579 \\
\hline Total protein, mean (range) $\mathrm{g} / \mathrm{dl}$ & $5.2(3.2-6.7)$ & $5.2(3.8-6.5)$ & 0.725 \\
\hline Älbumin, mean (range) g/dl & $2.8(1.6-3.9)$ & $2.6(1.4-3.5)$ & 0.907 \\
\hline Cholinesterase, mean (range) U/I & $163.4(70.0-306.0)$ & $156.2(38.0-262.0)$ & 0.640 \\
\hline Lymphocyte count, mean (range) /ml & $560.4(221.0-1,292.0)$ & $1,509.5(200.0-8,160.0)$ & 0.029 \\
\hline NLR, mean (range) & $8.5(3.2-21.3)$ & $9.8(1.9-23.3)$ & 0.668 \\
\hline Diameter of perforation, mean (range) $\mathrm{mm}$ & $12.1(10.0-15.0)$ & $3.8(0.0-5.0)$ & 0,046 \\
\hline Postoperative ICU stay, mean (range) days & $5.3(2.0-17.0)$ & $1.7(1.0-2.0)$ & 0,022 \\
\hline
\end{tabular}


rounded shape, more than $1 \mathrm{~cm}$ in diameter; (2) The colon is full of stool, which diffuses into the abdominal cavity through the perforation; (3) Ischemia and necrosis of the colonic mucosa leads to a feculent ulcer and an acute inflammatory reaction surrounding the perforation site that can be seen in microscopical examination; and (4) External injury or other diseases, such as obstruction, tumors and diverticulosis, must be excluded. In this study, after pathological examination, seven patients in the spontaneous group satisfied the above criteria.

The most frequent location of perforation is opposite the mesenteric edge of the sigmoid colon and the recto-sigmoid colon (3). This phenomenon may be due to the special physiological and anatomical features of the sigmoid colon. There is no ramus anastomoticus between the lowest branch of the sigmoid arteries and the superior rectal artery, which causes physiological ischemia. When stiff stool goes through the sigmoid colon, the colonic wall is compressed, which obstructs the blood supply. The blood supply to the opposite side of the mesentery is poor. The stool is more likely to stay in the rectosigmoid colon because of the narrowed colonic cavity. In addition, the smooth muscle contracts, which leads to an increase in pressure within the colonic cavity (6-9).

Spontaneous colonic perforation is more frequent in the elderly, and the mean age of onset is greater than 60 . Approximately 61 to $81 \%$ of patients had a history of constipation $(2,4)$. In this study, the mean age of the spontaneous group was 79.0 (71-87), which was significantly older than that of the diverticulosis group, and all patients had a long history of chronic constipation.

The possibility of this disease should be taken into consideration in elderly patients who have chronic constipation and are bed-ridden for long periods of time (10). In these patients, nutritional factors, such as body mass index, serum total protein, albumin, and cholinesterase, are often found to be low; however, no significant difference in nutritional factors was identified between the spontaneous group and the diverticulosis group in this study. We addressed the relationship between neutrophil-to-lymphocyte ratio (NLR) and perforation. The NLR is derived from the counts of circulating neutrophils and lymphocytes. It accurately represents the underlying inflammatory process (11). High NLR value was significantly associated with severe cholecystitis or appendicitis $(12,13)$. However, no significant difference in NLR value was identified between the spontaneous group and the diverticulosis group in this study.

Because the lymphocyte counts of the spontaneous group were significantly lower than those of the diverticulosis group, immune depression may be strongly associated with spontaneous perforation. However, a large-scale prospective or retrospective study is needed to clarify this issue.

\section{CONCLUSION}

In conclusion, spontaneous perforation of the sigmoid colon due to chronic constipation may not be due to undernutrition but instead due to immune depression.

\section{Conflict of Interest Statement}

The authors declare that there are no conflicts of interest regarding this study.

\section{REFERENCES}

1. Berry J.Dilatation and rupture of the sigmoid flexure. Br Med J 1984;1:301-302.

2. Serpell JW, Nicholls RJ. Stercoral perforation of the colon. Br J Surg 1990;77(12):1325-9.

3. Maurer CA, Renzulli P, Mazzucchelli L, Egger B, Seiler CA, Buchler MW. Use of accurate diagnostic criteria may increase incidence of stercoral perforation of the colon. Dis Colon Rectum 2000;43(7): 991-8.

4. Haddad R, Bursle G, Piper B. Stercoral perforation of the sigmoid colon. ANZ J Surg 2005;75(4):244-6.

5. Tokunaga Y, Hata K, Nishitai R, Kaganoi J, Nanbu H, Ohsumi K. Spontaneous perforation of the rectum with possible stercoral etiology: report of a case and review of the literature. Surg Today 1998;28(9):937-9.

6. Lui RC, Herz B, Plantilla E, Davidson AL, Cunningham JN Jr. Stercoral perforation of the colon: report of a new location. Am J Gastroenterol. 1988;83(4):457-9.

7. Matsuo S, Eguchi S, Azuma T, Kanetaka K, Itoh S, Yamaguchi S, Obata S, Kanematsu T. An unusual perforation of the colon: report of two cases. Surg Today 2002;32(9):836-9.

8. Huang WS, Wang CS, Hsieh CC, Lin PY, Chin CC, Wang JY. Management of patients with stercoral perforation of the sigmoid colon: report of five cases. World J Gastroenterol 2006;12(3):500-3.

9. Chakravartty S, Chang A, Nunoo-Mensah J. A systematic review of stercoral perforation. Colorectal Dis. 2013;15(8):930-5;4:58-59.

10. Yang B, Ni HK. Diagnosis and treatment of spontaneous colonic perforation: analysis of 10 cases. World J Gastroenterol 2008; 14(28):4569-72.

11. Tamhane UU, Aneja S, Montgomery D, Rogers EK, Eagle KA, Gurm HS: Association between admission neutrophil to lymphocyte ratio and outcomes in patients with acute coronary syndrome. Am J Cardiol 2008;102(6):653-7.

12. Lee SK, Lee SC, Park JW, Kim SJ. The utility of the preoperative neutrophil-to-lymphocyte ratio in predicting severe cholecystitis: a retrospective cohort study. BMC Surg 2014;14:100-6.

13. Yardımcı S, Ugurlu MÜ, Coskun M, Attaallah W, Yegen SC. Neutrophil-lymphocyte ratio and mean platelet volume can be a predictor for severity of acute appendicitis. Ulus Travma Acil Cerrahi Derg 2016;22(2):163-8. 